\title{
Análisis de la lluvia polínica actual en un gradiente altitudinal en los Andes de Chile Central ( $\left.33^{\circ} \mathrm{S}\right)$
}

\section{An analysis of modern pollen rain on an elevational gradient in the High-Andes of Central

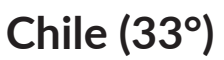

\author{
María del Pilar Fernández Murillo¹, Jaime G. Cuevas , $^{1,2,5}$ \& Antonio Maldonado ${ }^{1,3,4 *}$ \\ ${ }^{1}$ Centro de Estudios Avanzados en Zonas Áridas (CEAZA), Raúl Bitrán 1305, La Serena, Región de Coquimbo, Chile. \\ ${ }^{2}$ Centro de Investigación en Suelos Volcánicos (CISVo), Universidad Austral de Chile, Valdivia. \\ ${ }^{3}$ Instituto de Investigación Multidisciplinario en Ciencia y Tecnología, Universidad de La Serena. \\ ${ }^{4}$ Departamento de Biología Marina, Universidad Católica del Norte, Chile. \\ ${ }^{5}$ Instituto de Ecología y Biodiversidad (IEB), Santiago, Chile. \\ *Email: antonio.maldonado@ceaza.cl
}

\section{RESUMEN}

Los registros polínicos superficiales pueden ser una herramienta para caracterizar la vegetación de una determinada zona $y$, al igual que los registros de vegetación establecida, son aplicables para clasificar formaciones vegetales y determinar su diversidad. Aunque algunos estudios muestran la relación polenvegetación en Chile, pocos han explorado la diversidad polínica según gradientes altitudinales y la sensibilidad del polen como marcador de pisos vegetales. Mediante identificación morfológica, análisis de conglomerados y zonificación, se emplearon muestras de polen superficial para determinar grupos polínicos representativos de distintos niveles altitudinales y relacionar estas entidades con pisos vegetales ya establecidos. Adicionalmente, en tres cuencas de los Andes de Chile central, comparamos la diversidad polínica entre dichos pisos, como una medida integradora de la composición y abundancia del polen. En la cuenca de Laguna del Viento, cuatro grupos polínicos coincidieron con los pisos vegetales previamente definidos en la literatura: subandino, andino y altoandino, el último de los cuales fue subdividido en dos grupos. En la cuenca de El Yeso se determinaron dos grupos polínicos, todos correspondientes al piso andino. Finalmente, en la cuenca de El Volcán se registraron dos grupos polínicos, relacionados con los pisos vegetales subandino y andino. Solamente en una cuenca la diversidad polínica disminuyó con la altitud. Nuestros resultados muestran que la sensibilidad polínica permite diferenciar pisos vegetales, pero con una menor resolución que la brindada por los estudios vegetales clásicos. Esto se debe, probablemente, a factores diferenciales como la resolución taxonómica y la producción y dispersión del polen.

Palabras clave: Andes de Chile Central, distribución vegetal, diversidad, gradiente altitudinal, polen.

\section{ABSTRACT}

The superficial pollen records are tools for the vegetal reconstruction of a certain area. Like a plant record, pollen can be used to classify plant formations and determine plant diversity. Although some studies show the relationship between pollen and vegetation in Chile, few have explored the pollen diversity in an altitudinal gradient and its sensitivity as a marker of altitudinal belts. Consequently, we used pollen samples of surface soil and by means of morphological identification, cluster analysis and zonation we determined the pollen groups that represent the vegetation floors in an altitudinal gradient. Moreover, we compared pollen diversity among these floors for three basins of the Chilean Central Andes as a comprehensive index of the pollen composition and abundance. In the Laguna del Viento basin, four pollen groups coincided with the plant floors previously 
defined in the literature: sub Andean, Andean, and High Andean. The latter one was subdivided into two groups. In the El Yeso basin, two pollen groups were determined, all corresponding to the Andean floor, and finally in the El Volcán basin, two pollen groups were recorded that are related to the sub Andean and Andean vegetation floors. On the other hand, only in one basin the pollen diversity decreased with the altitude. These results showed that pollen assemblages can distinguish plant floors, but with a lower resolution than when using established flora, probably due to the pollen taxonomic resolution, differential production and dispersal factors.

Keywords: Altitude, Andes of Central Chile, diversity, pollen, vegetal distribution.

\section{INTRODUCCIÓN}

Los ecosistemas están en permanente interacción y cambio (Solomon et al. 2008), por lo que se pueden considerar como unidades inestables en el tiempo. Para comprender su dinámica presente y futura es fundamental conocer su historia, siendo la vegetación, en ese sentido, una de las fuentes más valiosas de información (Lagos 2017). Sin embargo, la cobertura y composición vegetal de muchos ecosistemas varía estacionalmente, lo que puede suponer una limitación a los estudios de vegetación (Cuevas \& Le Quesne 2006). Uno de los métodos indirectos para caracterizar la flora y vegetación de un sitio es el análisis de lluvia polínica, entendida como el conjunto de polen producido por la vegetación de un área determinada (Jackson \& Williams 2004). Esta aproximación representa una herramienta de estudio alternativa y complementaria a las investigaciones botánicas clásicas.

Por varias de sus características, el polen constituye una buena herramienta para caracterizar la vegetación. Por ejemplo, está disponible en el suelo de forma permanente, permite considerar diferentes escalas espaciales y estacionales (e.g. plantas anuales vs. perennes) (Kuentz et al. 2007) y, debido a su alta capacidad de preservación, es aplicable en áreas de investigación como la reconstrucción climática o de cambios en la vegetación del pasado (Trivi de Mandri et al. 2006, Gosling et al. 2009, Kuoppamaa et al. 2009). Numerosos trabajos han relacionado los registros de lluvia polínica con la distribución de flora actual, a fin de contar con una herramienta para mejorar la interpretación de registros de polen fósil (Heusser 1971, Ashworth et al. 1991, Páez et al. 1994, 2001, Lupo 1998, Weng et al. 2004, Reese \& Liu 2005, Cañellas-Boltà et al. 2009, Felde et al. 2016, Lagos 2017). Otras contribuciones, no obstante, sugieren que la relación polen-vegetación tiene ciertas limitaciones, atribuibles a tres factores principales: resolución taxonómica, dispersión y producción del polen (Oswald et al. 2003, Sugita et al. 2010, Meltsov et al. 2011). Respecto al primero, en la mayoría de los estudios sólo se pueden identificar entidades a nivel de familia, género o tipo polínico (Moore et al. 1991), salvo las familias monotípicas, en que sí se pueden reconocer especies. En cuanto al segundo, dado que el polen siempre tiene algún grado de dispersión antes de depositarse en el suelo, la cantidad de granos presentes en las muestras tiende a ser variable de un taxa a otro. Existen tipos polínicos que se pueden dispersar largas distancias y depositar en lugares donde la especie productora no se encuentra, a diferencia de otros cuya dispersión es más restringida. El tercero de los factores requiere considerar que algunos taxa (e.g. anemófilos) producen más polen que otros (e.g. entomófilos), razón por la cual la representatividad de las muestras polínicas de suelo puede variar de manera importante (Hidalgo et al. 2003, Gómez-Casero et al. 2004, Muñoz \& Arroyo 2006).

La mayoría de los trabajos sobre lluvia de polen actual relacionan registros polínicos con descripciones regionales de vegetación (Mancini 1998, Heusser et al. 1999, Haberle \& Bennett 2001, Páez et al. 2001, Heusser 2003, Weng et al. 2004). Sin embargo, aquellos que analizan la relación polen-vegetación de un área determinada y discuten sobre su representatividad son relativamente escasos (Villagrán 1980, Ashworth et al. 1991, Páez et al. 1997, Maldonado et al. 2005, Collao-Alvarado et al. 2015). En general, los trabajos de este tipo realizados en Chile están concentrados en el sur del país, principalmente en zonas de bosques (Villagrán 1980, Ashworth et al. 1991, Páez et al. 1997, Markgraf et al. 2002, Heusser 2003, Montade et al. 2019), y unos pocos en la zona altiplánica (Maldonado et al. 2005, González-Silvestre et al. 2013, Collao-Alvarado et al. 2015).

La Cordillera de los Andes mediterráneos es un territorio interesante para comparar diversidad polínica según gradientes altitudinales, desde una escala regional hasta una local, labor que aún no ha sido emprendida. Una singularidad de los Andes mediterráneos de Chile está dada por el alto grado de endemismo de su flora, debido a la presencia de grandes elevaciones que actúan como una barrera biogeográfica, que evita la migración de elementos florísticos desde y hacia Argentina (Smith-Ramírez et al. 2005, Arroyo et al. 2006). Además, la topografía montañosa (pendientes y exposiciones) crea condiciones climáticas locales y una alta 
heterogeneidad edáfica, que condicionan la vegetación de la zona (Luebert \& Pliscoff 2006, Luzio et al. 2010, Sarricolea et al. 2017). Los Andes también se caracterizan por presentar fuertes gradientes altitudinales, latitudinales y longitudinales, que contribuyen en forma adicional a la heterogeneidad de su flora y vegetación. Así, se espera la existencia de diversos pisos vegetales (Luebert \& Pliscoff 2006), los cuales ya han sido descritos por diversos autores (Squeo et al. 1993, Cavieres et al. 2000, Muñoz-Schick et al. 2000). La caracterización de dichos pisos altitudinales se ha basado esencialmente en la fisonomía de la vegetación establecida, aunque también se han introducido herramientas estadísticas que toman ventaja de la composición y abundancia de la flora (Cavieres et al. 2000). Sin embargo, ningún trabajo ha determinado la representatividad polínica de los pisos vegetales previamente definidos en los Andes de Chile central. El objetivo del presente artículo es determinar la diversidad polínica en un gradiente altitudinal y la correspondencia de esta con los pisos vegetales ya establecidos en tres cuencas hidrográficas de los Andes de Chile central. Dado que los conteos de polen también arrojan datos de composición y abundancia de tipos polínicos, considerando las limitaciones anteriormente citadas, se puede poner a prueba su contribución en la delimitación estadística de los pisos de vegetación. La importancia de este estudio radica en que permite analizar la zonación vegetal de un área, aun cuando las especies no estén presentes por variaciones estacionales, o bien estén cubiertas de nieve, dándonos cuenta de una forma alternativa y complementaria para caracterizar la vegetación, así mismo caracterizar la señal polínica de los distintos pisos de vegetación.

\section{MATERIALES Y MÉTODOS}

\section{Área de ESTUdio}

La zona cordillerana de la Región Metropolitana de Santiago en Chile Central $\left(33^{\circ}-34^{\circ} \mathrm{S}\right)$ limita al norte con las cumbres que separan la cuenca del Río Aconcagua de la cuenca del Río Maipo (Fig. 1), al sur con las cumbres que separan la cuenca del Río Maipo de la cuenca del Río Cachapoal y al oriente con Argentina. Sus elevaciones representativas son el cerro Marmolejo $(6.104 \mathrm{~m})$ y los volcanes Tupungato $(6.635 \mathrm{~m})$, Tupungatito $(5.650 \mathrm{~m})$, San José $(5.856 \mathrm{~m})$ y Maipo (5.264 $\mathrm{m})$. Los cursos fluviales más destacados del área son los ríos Colina, Olivares, Colorado, Yeso, Volcán, Mapocho y Maipo.

\section{Clima de LA zona}

El clima característico de esta zona corresponde al mediterráneo y presenta una alta variabilidad interanual de precipitaciones (Aceituno \& Vidal 1990). En zonas bajas, menores a $3.000 \mathrm{~m}$ s. n. m., se clasifica como clima templado mediterráneo con estación seca prolongada (Di Castri \& Hajek 1976, Sarricolea et al. 2017). La duración de dicha estación es de 4 a 5 meses y el invierno es bien marcado, con temperaturas extremas que llegan a $0^{\circ} \mathrm{C}$. En un transecto en los Andes de la Región Metropolitana de Santiago, Cavieres \& Arroyo (1999) midieron temperaturas medias del aire de $6.6^{\circ} \mathrm{C}$ a $2.600 \mathrm{~m}$ s. n. m. y de $3.0^{\circ} \mathrm{C}$ a $3.150 \mathrm{~m} \mathrm{~s}$. n. m. Las precipitaciones ocurren principalmente en las temporadas invernales y en los meses más lluviosos alcanzan entre 80 y 105 mm sobre 3.500 $\mathrm{m}$ de altitud (datos obtenidos de WORLDCLIM, resolución 30 seg, http://www.worldclim.org). En zonas altas, sobre 3.000 m s. n. m., el clima es clasificado como de tundra con verano seco (Sarricolea et al. 2017), en que las bajas temperaturas y las precipitaciones sólidas permiten la acumulación de nieve y campos de hielo permanente en cumbres y quebradas de la alta cordillera. La cobertura de nieve se mantiene de 3 a 5 meses, dependiendo de la elevación y la exposición solar (Rozzi et al. 1989, Cavieres et al. 2000).

\section{VEGETACIÓN DE LA ZONA}

Existen diversas clasificaciones de pisos de vegetación para los Andes de la Región Metropolitana de Chile. Por ejemplo, Quintanilla (1980) definió dos pisos, mientras que Arroyo et al. (1981) identificaron tres unidades altitudinales en la misma área. Teillier et al. (1994), en el Parque Nacional El Morado, definieron también tres pisos altitudinales, los que no coinciden exactamente con los descritos por los otros autores, tanto en denominación como en rango altitudinal. Aun permitiendo cierta variación geográfica en función de la latitud y longitud de los sitios estudiados, existe un componente de subjetividad inherente a las evaluaciones fisonómicas (Cavieres et al. 2000). Por ello, estos últimos autores reestudiaron la zonación vegetacional en los Andes de Santiago y llegaron a identificar seis a siete grupos basándose en métodos estadísticos cuantitativos que consideraban la composición y abundancia de la flora.

Para este estudio se consideró como punto de partida la división fisonómica de Teillier et al. (2011) y una subdivisión de acuerdo con lo propuesto por Luebert \& Pliscoff (2006) (Figs. 1 y 5). Si bien existen clasificaciones más detalladas, como las de Cavieres et al. (2000), esta no considera el nivel subandino (bajo $2.100 \mathrm{~m} \mathrm{s.} \mathrm{n.} \mathrm{m.),} \mathrm{a} \mathrm{diferencia} \mathrm{de} \mathrm{las}$ anteriores. No obstante, en la sección de Discusión se hace mención tanto a las clasificaciones fisonómicas como a las cuantitativas. De esta manera, para el área de estudio se han considerado cuatro diferentes pisos vegetales. En cuanto a la nomenclatura botánica, se sigue a Rodríguez et al. (2018).

En la zona de estudio, el límite superior de la flora vascular se sitúa alrededor de los $4.000 \mathrm{~m}$ de altitud, pese a que es 
posible encontrar ejemplares aislados de Nassauvia lagascae var. lagascae hasta $4.300 \mathrm{~m} \mathrm{s.} \mathrm{n.} \mathrm{m.,} \mathrm{los} \mathrm{cuales} \mathrm{crecen} \mathrm{entre}$ rocas en las faldas del volcán San José (Cuevas 1996). Por otra parte, cada tipo polínico registrado en el análisis fue asignado a algún piso de vegetación de los descritos por Teillier et al. (2011):

Piso subandino: Comprende desde los 1.800 a los $2.100 \mathrm{~m}$ s.n.m. Las comunidades vegetales de la zona son: a) Bosque esclerófilo mediterráneo andino de Kageneckia angustifolia D. Don y Guindilia trinervis Gillies ex Hook. \& Arn., que se caracteriza por presentar elementos de transición entre bosque y matorral, donde las especies arbóreas están representadas por K. angustifolia y por arbustivas dominantes como G. trinervis y Colliguaja integerrima Gillies \& Hook. En la comunidad de herbáceas se destacan Acaena pinnatifida Ruiz \& Pav. y Stachys grandidentata Lindl. b) Matorral espinoso mediterráneo interior de Trevoa quinquinervia Gillies \& Hook. y Colliguaja odorifera Molina. Esta comunidad tiene como especies dominantes las mencionadas anteriormente y Schinus polygamus (Cav.) Cabrera, con presencia en las partes altas de especies del bosque esclerófilo como Quillaja saponaria Molina, Lithrea caustica (Molina) Hook. \& Arn. y Kagenekia angustifolia D. Don., entre otros (Luebert \& Pliscoff 2006). Si bien esta última comunidad está bajo el nivel del piso subandino estudiado, consideramos importante incluirla en esta descripción debido a la posibilidad de registrar tipos polínicos por efecto de su dispersión.
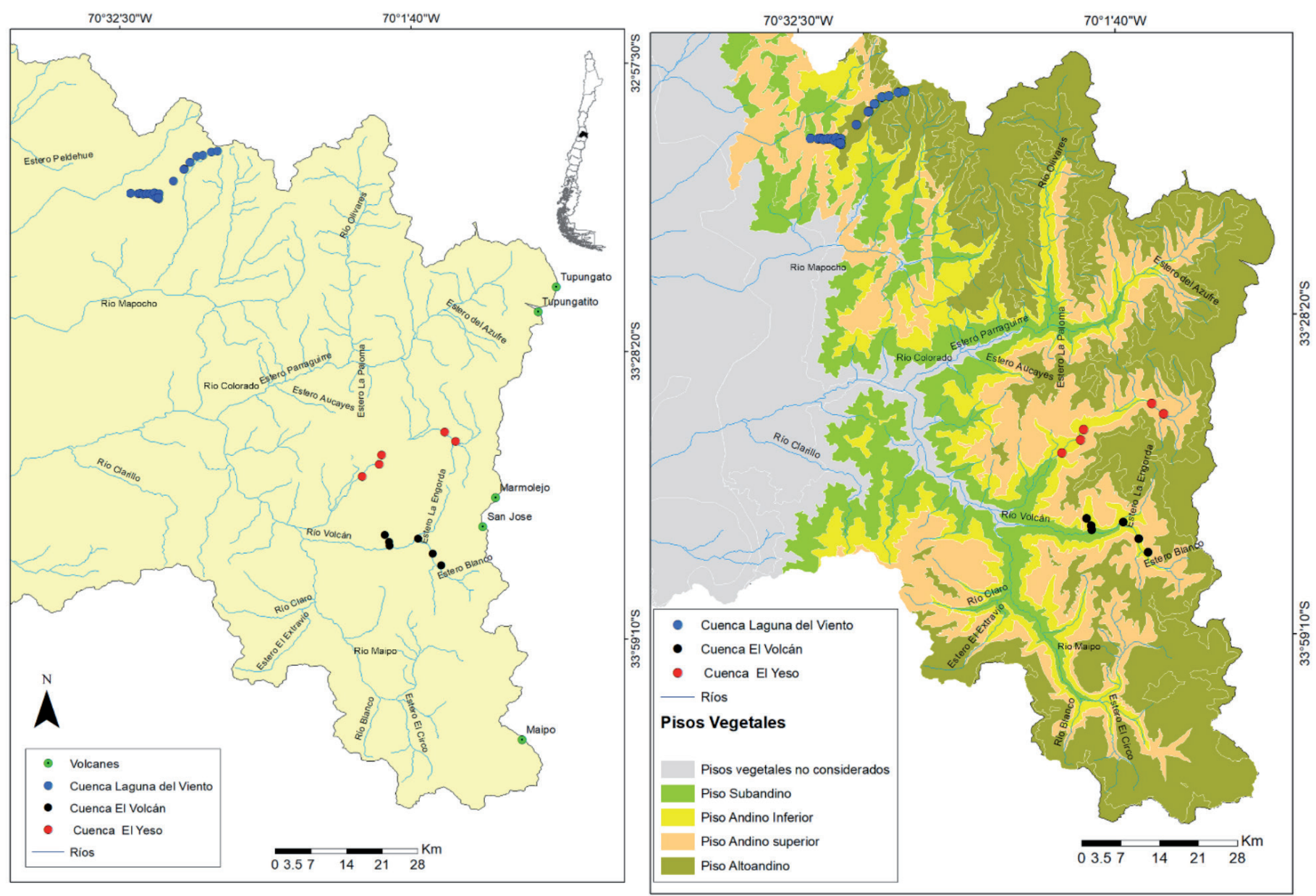

Figura 1. Izquierda: Mapa de ubicación de transectos en las tres cuencas Laguna del Viento, El Volcán y El Yeso, en la Región Metropolitana de Santiago. Los puntos de colores indican los sitios de muestreo. Derecha: Mapa de formaciones vegetales en base a Luebert \& Pliscoff (2006) y Teillier et al. (2011) para cada transecto. / Left: Map of transects in the three locations Laguna del Viento, El Volcán and El Yeso in the Región Metropolitana de Santiago. The colored dots indicate the sampling sites. Right: Map of vegetation zones based on Luebert \& Pliscoff (2006) and Teillier et al. (2011). 
Piso andino: Comprende la zona desde los 2.100 a los 3.300 m s. n. m. Este piso se caracteriza principalmente por una comunidad vegetal denominada matorral bajo mediterráneo andino (Luebert \& Pliscoff 2006). Según la dominancia de algunas especies, puede subdividirse en dos:

a. Piso andino inferior: Abarca la zona comprendida entre 2.100 y 2.800 m s. n. m. y su vegetación característica es el matorral bajo mediterráneo andino de Chuquiraga oppositifolia D. Don y Nardophyllum lanatum (Meyen) Cabrera. Esta comunidad está dominada por los arbustos ya mencionados, además de Azorella prolifera (Cav.) G.M. Plunkett \& A.N. Nicolas, Viviania marifolia Cav., Ephedra chilensis y Tetraglochin alatum (Gillies ex Hook. \& Arn.) Kuntze. Las herbáceas dominantes son Phacelia secunda J.F. Gmel. y Argylia adscendens DC. (Luebert \& Pliscoff 2006).

b. Piso andino superior: Comprende desde 2.800 hasta $3.300 \mathrm{~m}$ s. n. m. La comunidad vegetal distintiva de este piso es el matorral bajo mediterráneo andino de Azorella ruizii G.M. Plunkett \& A.N. Nicolas y Berberis empetrifolia Lam. y se caracteriza principalmente por la existencia de cojines y baja presencia de arbustos. Las especies más abundantes, además de las mencionadas, son: Acaena pinnatifida, Adesmia aegiceras Phil., Azorella madreporica Clos, Doniophyton weddellii Katinas \& Stuessy y Mutisia sinuata Cav. Entre las herbáceas destaca Oxalis compacta Gillies ex Hook. \& Arn. y diversas especies de la familia Poaceae (Luebert \& Pliscoff 2006).

Piso altoandino: Comprende desde 3.300 hasta los 4.000 m s. n. m. La vegetación es escasa, muy fragmentada, y con frecuencia las plantas crecen refugiadas entre las rocas. La comunidad vegetal de este piso corresponde al herbazal mediterráneo andino de Nastanthus scapigerus (J. Remy) Miers y Menonvillea spathulata (Gillies \& Hook.) Rollins, cuya forma de crecimiento principal es en roseta. Entre las especies destacadas figuran, aparte de las dos ya mencionadas, Nassauvia lagascae (D. Don) F. Meigen, Azorella trifurcata (Gaertn.) Pers., Deyeuxia erythrostachya E. Desv., Erigeron andicola DC., Leucheria scrobiculata D. Don, Oxalis erythrorhiza Gillies ex Hook. \& Arn. y Senecio lithostaurus Cabrera (Luebert \& Pliscoff 2006).

\section{Cuencas ANALIZADAS}

El trabajo se realizó a lo largo de tres cuencas ubicadas en la Región Metropolitana. La primera, denominada Laguna del Viento (LDV), está ubicada $42 \mathrm{~km}$ al noreste de Santiago y cuenta con un gradiente de muestreo que comprende rangos altitudinales de entre 1.800 ( $33^{\circ} 11^{\prime}$ 30,30" S; 70 31' 18,54"

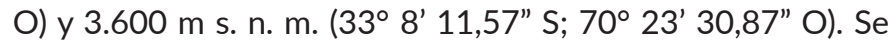
encuentra cerca del cordón andino de la Región de Valparaíso, Chile (Fig. 1). La segunda es la cuenca del Río Yeso (YE), que se encuentra ubicada $46 \mathrm{~km}$ al sureste de Santiago y cuyo gradiente altitudinal de muestreo abarca desde $2.100\left(33^{\circ} 41^{\prime}\right.$

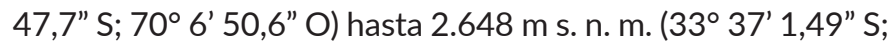
$69^{\circ} 58^{\prime} 8,08^{\prime \prime} \mathrm{O}$ ) (Fig. 1). La tercera de las cuencas corresponde a la del río Volcán (VO), que se ubica $52 \mathrm{~km}$ al sureste de Santiago y comprende rangos altitudinales de muestreo entre 1.900 ( $33^{\circ} 49^{\prime} 13,7^{\prime \prime}$ S; $70^{\circ} 3^{\prime} 56,77^{\prime \prime}$ O) y 2.400 m s. n. m. (33 $50^{\prime} 58,98^{\prime \prime}$ S; $69^{\circ} 59^{\prime}$ 5,53" O) (Fig. 1). Las dos últimas cuencas son afluentes del río Maipo, mientras que la primera tributa al río Mapocho antes de su unión al Maipo.

\section{MUESTREO Y PROCESAMIENTO}

En cada una de las cuencas estudiadas, cada $100 \mathrm{~m}$ de altitud aproximadamente, se realizaron muestreos de suelo en cinco puntos diferentes dentro de un área de $16 \mathrm{~m}^{2}$. En cada uno de los puntos se recolectaron muestras de alrededor de 10 a $20 \mathrm{~g}$ del primer centímetro de suelo, que representaría la vegetación presente de la zona (Adam \& Mehringer 1975). Para la extracción de polen se procesó $1 \mathrm{~cm}^{3}$ de suelo de cada muestra compuesta. El tratamiento consistió en agregar dos pastillas de esporas de Lycopodium clavatum para cálculos de concentración polínica (Stockmarr 1971), tras lo cual se realizaron tratamientos estándares para la extracción de polen, que incluyeron defloculación y eliminación de ácidos húmicos con $\mathrm{KOH}$ al 10\%, remoción de los carbonatos con $\mathrm{HCl}$ al $10 \%$, remoción de silicatos con HF y posterior acetólisis para eliminar la materia orgánica (Faegri \& Iversen 1989). Posteriormente, se almacenó el extracto obtenido en glicerina. Se tomó una alícuota de dicho extracto, a partir de la cual se efectuó un recuento igual o mayor a $\mathbf{3 0 0}$ granos de polen por muestra, utilizando microscopios de luz con aumento de 400 x y 1.000 x. Los cálculos de porcentaje polínico fueron hechos sobre una suma básica, que incluye todos los tipos polínicos registrados por cada nivel altitudinal, excluyendo taxa acuáticos y palustres en caso de que hubiera.

Los granos de polen se determinaron hasta nivel de género o familia utilizando para ello muestras de referencia de la región y descripciones bibliográficas (Heusser 1971, Markgraf \& D'Antoni 1978). Para apoyar estadísticamente la determinación de las zonas polínicas, se realizó un análisis de conglomerados con el método del incremento de suma de cuadrados (CONISS, Constrained Incremental Sums of Squares, Grimm 1987), utilizando el programa TILIA 1.7.16 (Grimm 1991-2011; Springfield, IL, USA).

Dicho análisis, de amplio uso en palinología, permite agrupar las muestras con mayor homogeneidad en un grupo polínico determinado y distinguirlas de otros con mayores diferencias, pero respetando el orden altitudinal de estos y considerando 
el significado ecológico. De no respetarse el orden altitudinal, pueden generarse agrupaciones entre niveles que no son contiguos, perdiéndose así la correspondencia entre la situación real y la separación estadística. Los datos fueron previamente transformados con la función raíz cuadrada, lo que permite compensar por las diferencias de abundancia entre distintos taxa. Solamente se consideraron aquellos taxa que por lo menos en una muestra tuvieran abundancias mayores o iguales a $3 \%$, con objeto de depurar la base de datos del ruido asociado a bajas abundancias (Bennett 1996). La delimitación de zonas estadísticamente significativas se hizo con el programa psimpoll (Bennett 1996), que está basado en el modelo de la rama rota (broken-stick model).

Para efectos de representación gráfica, los taxa con una proporción inferior a $3 \%$ fueron agregados a un único grupo denominado "otros» (Figs. 2, 3, 4), dado que varios taxa sólo estuvieron representados por uno o dos granos de polen.

\section{DiVERSIDAD POLÍNICA}

Se determinó la diversidad polínica considerando los grupos altitudinales obtenidos en el análisis de conglomerados (CONISS) y la posterior zonificación según Bennett (1996). Para cada grupo se determinó el número de tipos polínicos y se calculó el índice de Shannon-Wiener $\left(H^{\prime}=-\Sigma p_{i}^{*} \ln \left(p_{i}\right)\right)$, en que $p_{i}$ es la proporción en abundancia del respectivo tipo polínico sobre el conteo total de 300 granos de polen y In es el logaritmo natural. Dicho índice, ampliamente utilizado en estudios de vegetación (Magurran 1988), fue calculado mediante el programa Past 2.0.

\section{RESULTADOS}

\section{1) Cuenca Laguna del Viento}

Este transecto se ubica en la comuna de Colina y comprende 18 puntos de muestreo, desde 1.800 a $3.522 \mathrm{~m}$ de altitud. Los primeros puntos de muestreo se encuentran cercanos al río principal, Colina, y los últimos continúan por un camino secundario (Fig. 1). En general, la concentración polínica en el transecto de Laguna del Viento no varió fuertemente entre niveles (Fig. 2). La concentración en el nivel más alto (3.522 m s. n. m.) fue de 280.555 .650 granos $/ \mathrm{cm}^{3}$ mientras que en el nivel más bajo ( $1.800 \mathrm{~m} \mathrm{s.} \mathrm{n.} \mathrm{m.)} \mathrm{fue} \mathrm{de} 211.463 .082$ granos/ $\mathrm{cm}^{3}$. El nivel con mayor concentración polínica correspondió a $3.175 \mathrm{~m} \mathrm{s.} \mathrm{n.} \mathrm{m.,} \mathrm{con} 600.000 .000$ granos $/ \mathrm{cm}^{3}$.

En cuanto a la composición polínica a través del transecto, las familias dominantes fueron Asteraceae, Poaceae, Caryophyllaceae, Euphorbiaceae y Ephedraceae. En la Figura 2 se presenta el diagrama de porcentajes polínicos. Según el análisis de conglomerados (CONISS) y la zonificación de psimpoll, las muestras fueron agrupadas en cuatro grupos, con delimitación de zonas polínicas en los niveles $2.350 \mathrm{~m} \mathrm{s.} \mathrm{n.} \mathrm{m.,}$ 3.275 m s. n. m. y 3.429 m s. n. m.

\section{GRUPO I (1.800-2.350 m s. n. m.)}

Este grupo se caracterizó por la dominancia de los taxa polínicos Asteraceae tipo Senecio y Asteraceae tipo Baccharis. En los niveles más bajos, 1.800-1.900 m s. n. m., se encontró una mayor representación de los taxa polínicos Asteraceae tipo Senecio (12\%), Asteraceae tipo Mutisia (10\%), Euphorbiaceae (15\%) y Asteraceae tipo Baccharis (42\%). Los niveles superiores de este grupo $(2.000-2.300 \mathrm{~m} \mathrm{~s}$. $\mathrm{n}$. m.) tuvieron como taxón más representativo a Asteraceae tipo Senecio (55\%). A diferencia de los niveles inferiores, en estos se registraron taxa como Brassicaceae, Apiaceae tipo Laretia, Euphorbiaceae tipo Colliguaja, Fabaceae tipo Adesmia y Rosaceae tipo Acaena, con porcentajes menores de $5 \%$.

\section{GRUPO II (2.350-3.275 m s. n. m.)}

Este grupo estuvo dominado por taxa polínicos como Poaceae, Caryophyllaceae tipo Cerastium, Asteraceae tipo Mutisia, Euphorbiaceae y Asteraceae tipo Senecio. El taxón Asteraceae tipo Baccharis estuvo menos representado que en el Grupo I, mientras que taxa como Ephedra, Solanaceae y Asteraceae tipo Leucheria tuvieron mayor representatividad en este grupo que en los otros.

En los niveles inferiores del grupo $(2.400-2.800 \mathrm{~m} \mathrm{~s}$. n. m.) se registró una mayor presencia de Solanaceae $(20 \%)$ y Ephedra (18\%). A partir de $2.700 \mathrm{~m}$ de altitud, se observó que Asteraceae tipo Senecio aumentó y Euphorbiaceae tipo Colliguaja alcanzó su valor máximo ( 15\%). En los niveles superiores del grupo, se destacó la presencia de Caryophyllaceae tipo Cerastium y un aumento en la proporción de Poaceae. Por su parte, Solanaceae y Euphorbiaceae tipo Colliguaja comenzaron a disminuir en relación con los niveles inferiores. El nivel superior de este grupo (3.175 m s. n. m.) presentó la mayor concentración polínica de todo el transecto y destacó por la presencia de la familia Rubiaceae, ausente en los otros niveles.

\section{GRUPO III (3.275-3.429 m s. n. m.)}

Este grupo mostró una dominancia de los taxa polínicos Asteraceae tipo Leucheria (hasta $40 \%$ ) y Poaceae (12\%) y una disminución de Ephedra (3\%). Además, se advirtió la aparición de nuevos taxa polínicos, como Plantago y Oxalis, ambos con porcentajes menores de $10 \%$.

\section{GRUPO IV (3.429-3.522 m s. n. m.)}

Este grupo estuvo dominado por los taxa polínicos Poaceae (35-40\%), Apiaceae tipo Pozoa (mejor representado en el nivel superior, con un 15\%), Ephedra (15-7\%) y Asteraceae tipo Senecio ( 10\%). Taxa polínicos como Asteraceae tipo Conyza, Caryophyllaceae tipo Pycnophyllum y Verbenaceae tipo Junellia fueron más abundantes en este grupo que en los otros. 


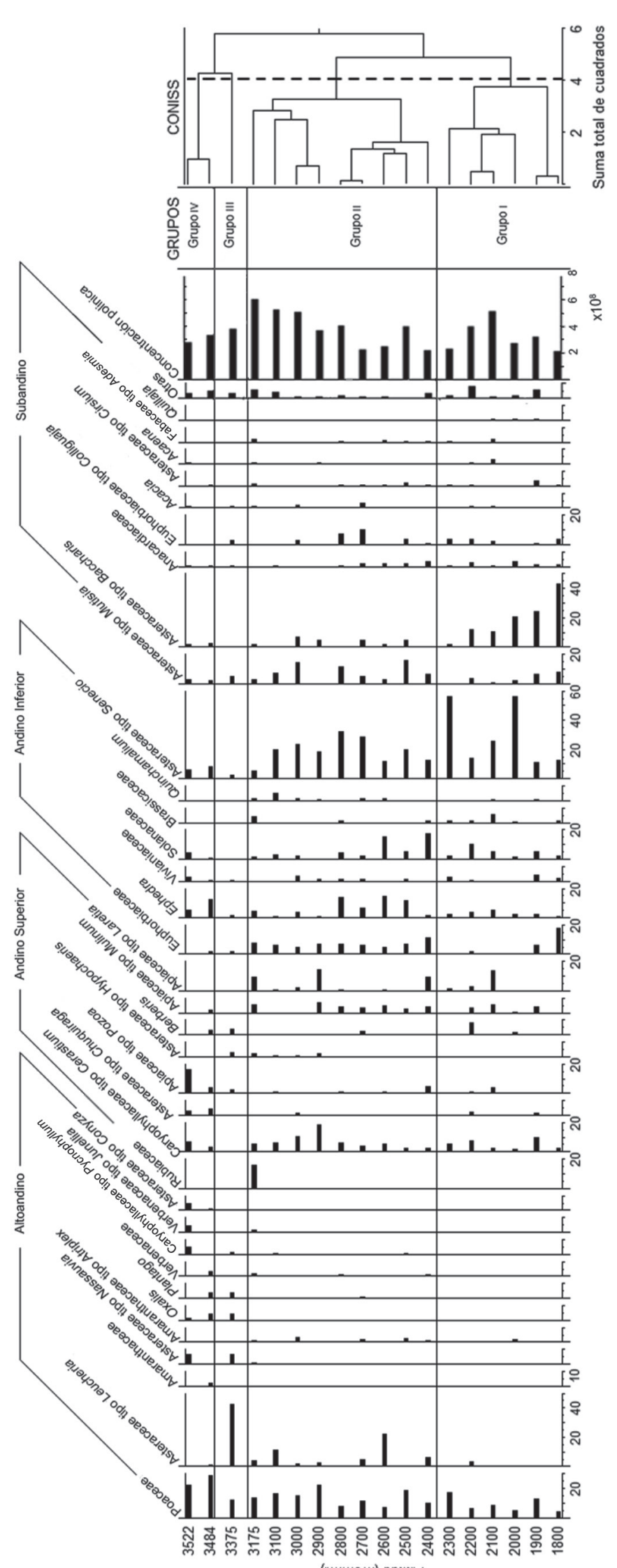

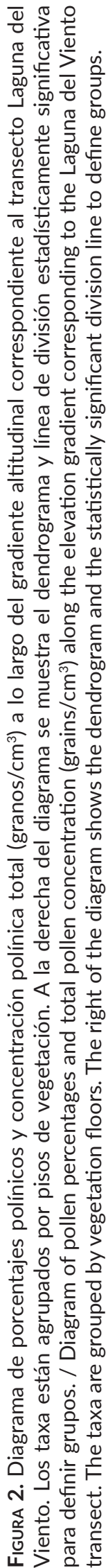




\section{2) Cuenca Río Yeso}

Este transecto se sitúa en la comuna de San José de Maipo y sigue el curso del río Yeso. Comprende cinco puntos de muestreo, ubicados cerca del embalse El Yeso entre 2100 y 2.648 m s. n. m. (Fig. 1). En los niveles $2.100,2.467$ y 2.537 $\mathrm{m} \mathrm{s}$. n. m, la concentración polínica presentó valores mayores de 600.000 .000 granos $/ \mathrm{cm}^{3}$. En cambio, los niveles 2.259 y $2.648 \mathrm{~m}$ s. n. m. mostraron concentraciones de alrededor de 400.000 .000 granos $/ \mathrm{cm}^{3}$ (Fig. 3, columna de concentración polínica).

Los taxa polínicos dominantes en el transecto fueron Poaceae, Ephedra y Asteraceae tipo Leucheria. En la Figura 3 se presenta el diagrama de porcentajes polínicos, cuyas muestras fueron distribuidas en dos grupos según el análisis de conglomerados (CONISS) y la zonificación de psimpoll. La división entre estos dos grupos se ubicó a 2.179 m s. n. m.

\section{GRUPO I (2100 m s. n. m.)}

Este grupo estuvo dominado por el taxón polínico Asteraceae tipo Leucheria, con un 58\%, acompañado de Poaceae (20\%) y Ephedra (7 \%).

\section{GRUPO II (2.179-2.648 m s. n. m.)}

Los taxa dominantes de este grupo fueron Ephedra (15-46\%), de mayor representación en los niveles inferiores, Poaceae (10-30\%) y Asteraceae tipo Leucheria (5-20\%). El nivel superior estuvo dominado por Asteraceae tipo Nardophyllum (hasta 32\%), que tuvo entre sus taxa acompañantes a Asteraceae tipo Leucheria, Ephedra, Poaceae y Asteraceae tipo Doniophyton.

\section{3) Cuenca Río Volcán}

Este transecto está ubicado en la comuna de San José de Maipo, sigue el curso del río Volcán y sus puntos de muestreo se encuentran próximos al estero La Engorda, quebrada del volcán Marmolejo y cerro Yesillo. Comprende desde 1.923 hasta $2.436 \mathrm{~m} \mathrm{~s}$. n. m. y cuenta con seis puntos de muestreo (Fig. 1). La concentración polínica de la cuenca de El Volcán mostró valores máximos en los niveles $2.078,2.391$ y $2.436 \mathrm{~m}$ s. n. m., con más de 500.000 .000 granos $/ \mathrm{cm}^{3}$. En cambio, los niveles 1.923 y $2.194 \mathrm{~m} \mathrm{s.} \mathrm{n.} \mathrm{m.} \mathrm{presentaron} \mathrm{concentraciones}$ polínicas menores de 300.000 .000 granos $/ \mathrm{cm}^{3}$ (Fig. 4).

Los taxa polínicos más representativos fueron Ephedra, Poaceae, Quinchamalium, Asteraceae tipo Taraxacum, Asteraceae tipo Mutisia y Apiaceae tipo Laretia. En la Fig. 4 se presentan el diagrama de polen en porcentajes, el análisis de conglomerados (CONISS) y la zonificación de psimpoll. Según este último análisis, es posible diferenciar dos grupos, separados a los $2.213 \mathrm{~m} \mathrm{s.} \mathrm{n.} \mathrm{m.,} \mathrm{a} \mathrm{saber:}$

\section{GRUPO I (1.923-2.213 m s. n. m.)}

Este grupo abarcó tres niveles. El inferior estuvo dominado por Poaceae (17-21\%); el superior, por Asteraceae tipo Taraxacum (10-20\%), y el central, por Asteraceae tipo Mutisia (hasta 40\%). Entre los taxa polínicos acompañantes figuraron Quinchamalium (hasta 16\%), Apiaceae tipo Pozoa (hasta 18\%) y Asteraceae tipo Leucheria (hasta 16\%).

\section{GRUPO II (2.213-2.436 m s. n. m.)}

El nivel inferior de este grupo estuvo ampliamente dominado por Ephedra (en torno a 60\%), mientras que en el superior el taxón dominante fue Apiaceae tipo Laretia, con valores de frecuencia de $60 \%$. Aunque en bajas proporciones, sólo en la parte superior de este grupo se registraron los taxa Asteraceae tipo Senecio (7\%) y Asteraceae tipo Conyza (5\%).

\section{RIQUEZA Y DIVERSIDAD POLÍNICA POR CUENCA}

La cuenca de Laguna del Viento presentó cerca de 49 taxa polínicos, de los cuales 10 corresponden a la familia Asteraceae y tres a Apiaceae. Los restantes 36 taxa pertenecen a otras familias, cada una de las cuales está representada por un tipo polínico. En la cuenca de El Yeso se identificaron 41 taxa polínicos; nueve corresponden a la familia Asteraceae, dos a Solanaceae, dos a Vivianiaceae y dos a Euphorbiaceae. Por lo que respecta a los 26 taxa restantes, se registró un tipo polínico por familia. En la cuenca del río El Volcán se registró un total de 40 taxa polínicos, 12 de la familia Asteraceae, dos de Apiaceae, dos de Amaranthaceae, dos de Euphorbiaceae y dos de Solanaceae. Los otros 20 taxa contaron con un tipo polínico por familia. En ninguna de las cuencas fue posible diferenciar tipos polínicos dentro de la familia Poaceae, factor que podría afectar la estimación de su riqueza y diversidad.

En la cuenca de Laguna del Viento, el Grupo II (2.350$3.275 \mathrm{~m} \mathrm{~s}$. n. m.) estuvo representado por 42 taxa polínicos (Tabla 1), el Grupo I (1.800-2.350 m s. n. m.) por 36, el Grupo III (3.275-3.429 m s. n. m.) por 22 y el Grupo IV (3.429-3.522 m s. n. m.) por 31. El índice de diversidad del Grupo II fue de 2,851 , valor comparativamente más alto que los obtenidos en los Grupos I, III y IV, cuyos índices fueron de 2,558, 2,213 y 2,826, respectivamente (Tabla 1).

En El Yeso, el análisis de diversidad polínica mostró que el Grupo I (2.100 m s. n. m.) contiene 14 tipos polínicos y un índice de diversidad de 1,44, mientras que el Grupo II (2.179$2.648 \mathrm{~m} \mathrm{s.} \mathrm{n.} \mathrm{m.)} \mathrm{presenta} 40$ tipos polínicos y un índice de diversidad de 2,33. Finalmente, el análisis de diversidad polínica de El Volcán mostró que el Grupo I (1.923-2.213 m s. n. m.) es el más diverso, con 36 taxa polínicos, en comparación con el Grupo II (2.213-2.436 m s. n. m.), que presentó 26 taxa polínicos. El índice de diversidad del Grupo I fue de 2,713, en tanto que el del Grupo II fue de 2,0 (Tabla 1). 

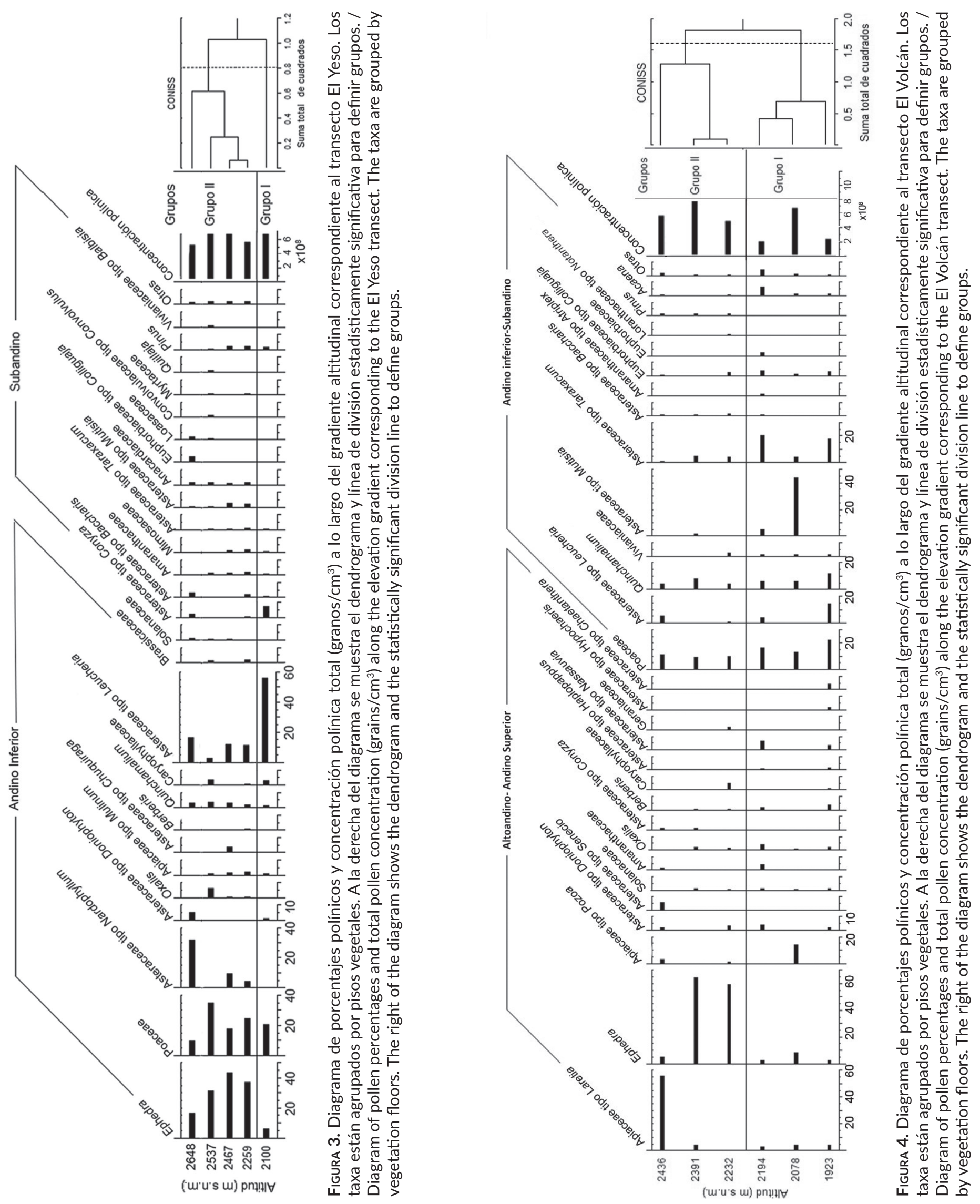
TABLA 1. Riqueza y diversidad de taxa polínicos ( $\left.H^{\prime}\right)$ registradas para las muestras de suelo en tres cuencas de la Región Metropolitana de Santiago. / Richness and diversity ( $\left.\mathrm{H}^{\prime}\right)$ of pollen taxa found in soil samples in the Región Metropolitana de Santiago.

\begin{tabular}{lcccc}
\hline Cuenca & Grupo & $\begin{array}{c}\text { Rango altitudinal } \\
\text { (m s.n.m.) }\end{array}$ & Riqueza polínica & $\begin{array}{c}\text { H' } \\
\text { (Shannon-Wiener) por grupo }\end{array}$ \\
\hline \multirow{3}{*}{ Laguna del Viento } & I & $1800-2350$ & 36 & 2,558 \\
& II & $2350-3275$ & 42 & 2,851 \\
& III & $3275-3429$ & 22 & 2,213 \\
& IV & $3429-3522$ & 31 & 2,826 \\
\hline El Yeso & I & 2100 & 14 & 1,440 \\
& II & $2179-2648$ & 40 & 2,330 \\
El Volcán & I & $1923-2213$ & 36 & 2,713 \\
\hline
\end{tabular}

Los grupos fueron determinados por análisis de conglomerados (CONISS) y por el método de la rama rota (Bennett 1996). / The groups were established through CLUSTER analysis (CONISS) and plotted in a dendrogram (Bennett, 1996).

\section{DISCUSIÓN}

\section{SEÑAL PoLínICA Y PISOS ALTITUdINALES de VEGetACIÓN}

Las agrupaciones de taxa polínicos en los distintos rangos altitudinales estuvieron dadas principalmente por la presencia y abundancia de los tipos de polen más frecuentes. Así, en el transecto de Laguna del Viento el Grupo I (1.800-2.350 m s. n. m.) presentó una mayor abundancia polínica de Asteraceae tipo Baccharis y Asteraceae tipo Senecio y su rango altitudinal coincidió en gran medida con los límites del piso subandino (Fig. 5). Según Teillier et al. (2011), el límite superior de este se encuentra a $2.100 \mathrm{~m} \mathrm{~s}$. n. m., mientras que Luebert \& Pliscoff (2006) lo extienden hasta $2.200 \mathrm{~m}$ de altitud. En este grupo se registró de manera exclusiva la presencia de Quillaja, que, por su hábito arbóreo siempreverde, sólo se desarrolla a baja altitud y es característico del piso subandino (Cruz et al. 2013). Este piso también se distingue por la presencia de varias especies de Baccharis, como B. neaei (DC.), B. poeppigiana (DC.), B. rhomboidalis (J. Remy) y B. sagittalis (Less) DC. (Teillier et al. 2011), las que no son diferenciables mediante rasgos polínicos.

El Grupo II (2.350-3.275 m s. n. m.) estuvo dominado por los taxa Asteraceae tipo Senecio, Ephedra, Euphorbiaceae y Caryophyllaceae tipo Cerastium, característicos del piso andino, que, según Teillier et al. (2011), puede dividirse en dos subgrupos: andino inferior (2.100-2.800 m s. n. m.) y andino superior (2.800-3.300 m s. n. m.). En el presente estudio, estos no se distinguieron estadísticamente, pero sí mediante el dendrograma CONISS, que mostró una separación de ambos pisos a $2.800 \mathrm{~m} \mathrm{s.} \mathrm{n.} \mathrm{m.,} \mathrm{caracterizado}$ por una mayor presencia de Poaceae, Caryophyllaceae tipo Cerastium y Apiaceae tipo Laretia y una disminución de
Solanaceae y Ephedra. El límite inferior del Grupo II fue 250 m más alto que el propuesto por Teillier et al. (2011) para el piso andino inferior; sin embargo, su límite superior coincidió con el señalado por dichos autores y Luebert \& Pliscoff (2006). Si bien no existe concordancia plena entre los límites observados y los reportados en la literatura, se registró una alta abundancia polínica de Ephedra, taxón característico del piso andino, y Colliguaja, representado en la flora actual de los pisos subandino y andino inferior. Los niveles superiores del grupo (2.900-3.175 m s. n. m.) estuvieron dominados por Asteraceae tipo Senecio y Poaceae y se caracterizaron, además, por una gran abundancia de Caryophyllaceae tipo Cerastium, taxón distintivo del piso andino superior. En el límite superior de este grupo, a los $3.175 \mathrm{~m}$ de altitud, se observó la presencia de Asteraceae tipo Nassauvia, lo que sugiere el inicio de un ambiente altoandino. Así mismo, el registro de Rubiaceae en este nivel permite inferir una transición entre los pisos andino superior y altoandino, pues dicha familia es muy representativa de este último piso.

Los grupos III (3.275-3.429 m s. n. m.) y IV (3.429-3.522 m s. n. m.) estuvieron dominados por Poaceae y Asteraceae tipo Leucheria (para el grupo III), taxa polínicos asignables a los pisos andino superior y altoandino. El Grupo IV fue el único en que se registraron los taxa polínicos Amaranthaceae y Verbenaceae tipo Junellia, entidades muy características del piso altoandino (Teillier et al. 2011). Según estos autores, el piso alto andino se encuentra entre los rangos 3.300 a 3.800 m s. n. m. Sin embargo, Cavieres et al. (2000) sugieren una subdivisión donde las altitudes $3.500,3.600$ y $3.700 \mathrm{~m} \mathrm{~s}$. n. $\mathrm{m}$. son independientes y no registran especies en común. Esta situación es muy parecida a la observada en nuestros datos (3.429 m s. n. m.). 
En la cuenca de El Yeso se registraron dos grupos: el Grupo I ( $2.100 \mathrm{~m} \mathrm{s.} \mathrm{n.} \mathrm{m.),} \mathrm{dominado} \mathrm{por} \mathrm{el} \mathrm{taxa} \mathrm{polínico} \mathrm{Asteraceae} \mathrm{tipo}$ Leucheria, y el Grupo II (2.179-2.648 m s. n. m.), caracterizado por una mayor abundancia de Poaceae y Ephedra y cuyo límite superior estuvo definido por una alta abundancia de Asteraceae tipo Nardophyllum. Aunque el análisis de conglomerados (CONISS) y el de psimpoll realizaron esta separación según la abundancia polínica, tanto por sus rangos altitudinales como por su presencia, todos los taxa registrados son característicos del piso andino y concuerdan con la descripción que Teillier et al. (2011) realizaron de este piso (Fig. 5).

Finalmente, en la cuenca del Río Volcán el Grupo I (1.923$2.213 \mathrm{~m} \mathrm{s.} \mathrm{n.} \mathrm{m.)} \mathrm{presentó} \mathrm{dominancia} \mathrm{de} \mathrm{Asteraceae} \mathrm{tipo}$ Mutisia, Poaceae, Asteraceae tipo Taraxacum y Quinchamalium, taxa polínicos comunes en el piso subandino. Según Teillier et al. (2011), este nivel comprende rangos altitudinales de entre 1.800 y $2.100 \mathrm{~m} \mathrm{~s}$. n. m. y se caracteriza por la abundancia de arbustos, en tanto que el andino inferior se extiende desde 2.100 hasta $2.800 \mathrm{~m}$ de altitud. Con base en esto, el grupo I correspondería a una transición del piso subandino al andino inferior. Por otra parte, el Grupo II (2.213-2.436 m s. n. m.), definido por la presencia de Apiaceae tipo Laretia, podría asociarse al piso andino superior (Fig. 5). El límite entre ambas zonas fue determinado a $2.213 \mathrm{~m} \mathrm{~s}$. n. m., resultado muy cercano a lo señalado por Teillier et al. (2011).
Al comparar los pisos altitudinales entre cuencas, fue posible distinguir una clara independencia entre estas. Todas las unidades presentaron taxa polínicos que definen pisos vegetales (señales polínicas), pero en niveles altitudinales equivalentes no compartieron necesariamente los mismos ensambles de polen. Por ejemplo, a 2.100 m s. n. m., los taxa polínicos dominantes en Laguna del Viento fueron Asteraceae tipo Senecio, Asteraceae tipo Baccharis, Solanaceae y Ephedra, mientras que en El Yeso fueron Asteraceae tipo Leucheria, Asteraceae tipo Conyza, Poaceae y Ephedra. En tanto, los taxa polínicos dominantes en El Volcán a $2.078 \mathrm{~m} \mathrm{~s}$. n. m. fueron Asteraceae tipo Mutisia y Apiaceae tipo Pozoa. Tal variedad de ensambles en un mismo nivel altitudinal, pero en diferentes cuencas, podría relacionarse con atributos intrínsecos de estas últimas, por ejemplo, la orientación de exposición al sol de cada cuenca, ya que cada una cuenta con un sistema físico y climático particular que hace que la vegetación varíe entre sitios y niveles (Teillier et al. 2011). Sin embargo, existen señales polínicas y una variación taxonómica entre grupos que permiten asignar a estos últimos a un determinado piso vegetal (Villagrán et al. 1983). Finalmente, debe considerarse que los límites inferior y superior de cada piso vegetal pueden variar entre cuencas, dependiendo de la exposición polar o ecuatorial de las laderas (Teillier et al. 2011).

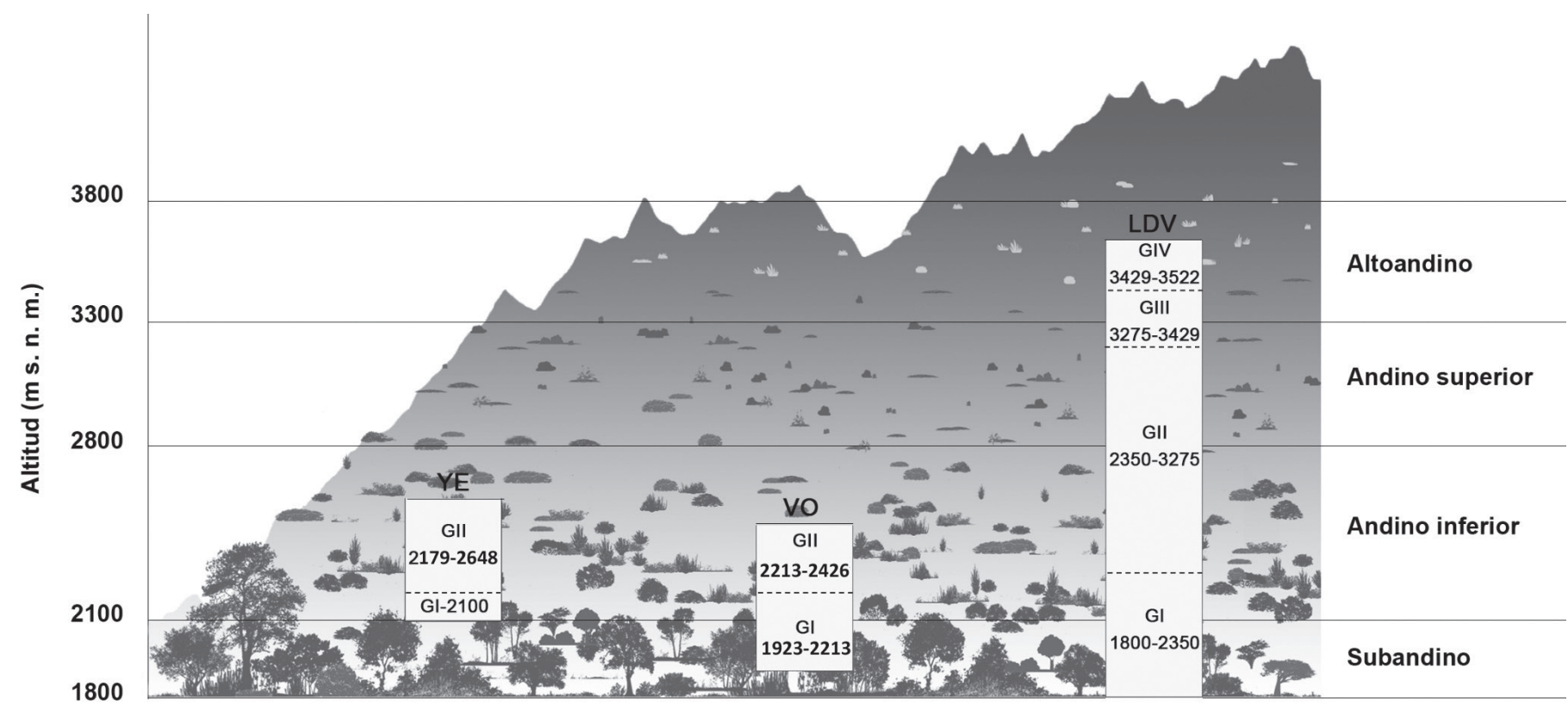

Fıgura 5. Pisos altitudinales de vegetación de acuerdo a Teillier et al. (2011) y su correspondencia con los grupos obtenidos en el análisis de polen. En el gráfico se indica el grupo (G) por cada cuenca y su rango altitudinal en m s.n.m. YE corresponde a la cuenca El Yeso, VO corresponde a la cuenca del Río Volcán y LDV corresponde a la cuenca de Laguna del Viento. / Vegetation belts in the elevational zones according to Teillier et al. (2011) and its correspondence with the groups obtained in the pollen analysis. In the graph the group (G) is indicated for each basin and its altitudinal range is in $\mathrm{m}$ a.s.l. YE corresponds to the El Yeso basin, VO corresponds to the Río Volcán basin and LDV corresponds to the Laguna del Viento basin. 


\section{RIQUEZA, DIVERSIDAD Y CONCENTRACIÓN POLÍNICA}

La diversidad polínica en el transecto de Laguna del Viento disminuyó tanto en los niveles 3.275- 3.429 m s. n. m. como en los de menor altitud (1.800-2.350 m s. n. m.). El Grupo II (2.3503.275 m s. n. m.) presentó una mayor riqueza y diversidad de taxa polínicos que los grupos I, III y IV. Por ser de transición, es posible que este grupo contenga una mezcla de taxa polínicos, en baja concentración, asignables a los pisos subandino y altoandino. Comparativamente, en la cuenca de El Volcán se observó una disminución más clara de la riqueza y diversidad polínica con el aumento de la altitud. Cavieres et al. (2000) (ver también Arroyo et al. 1988), en un área de la cordillera de Chile central muy próxima a nuestros sitios de estudio, determinaron que la riqueza vegetal disminuye a medida que aumenta la altitud y que el mayor número de especies se registra entre 2.500 y 2.700 m s. n. m. Dichos autores señalan, además, que la riqueza disminuye paulatinamente hasta llegar a una sola especie a los $3.700 \mathrm{~m} \mathrm{s.} \mathrm{n.} \mathrm{m.} \mathrm{Así,} \mathrm{nuestros} \mathrm{datos}$ muestran una tendencia de disminución de la diversidad similar a la obtenida con estudios de flora actual.

La tendencia correspondiente a El Yeso difiere de las observadas en Laguna del Viento y El Volcán, pues indica que la riqueza y diversidad polínica aumentan con el incremento de la altitud. Pese a tener rangos altitudinales limitados, la diversidad polínica en la cuenca de El Yeso incrementó en aproximadamente una unidad en el índice de ShannonWiener, al contrario de lo que se registró en Laguna del Viento que no tiene grandes variaciones, y en el Río Volcán en que la diversidad disminuye con el aumento de altitud en menor grado.

Cabe señalar que, pese a abarcar rangos altitudinales estrechos, los transectos de El Volcán y El Yeso presentan taxa polínicos únicos, tales como Boraginaceae tipo Heliotropium y Urticaceae tipo Urtica, respectivamente, lo que resulta atribuible al efecto de las quebradas (ver más abajo). Dichos taxa fueron incorporados al grupo «otros» por presentar porcentajes menores a $3 \%$.

El índice de Shannon-Wiener calculado en este trabajo tiene una relación con la riqueza taxonómica y abundancia de los granos de polen. Este índice nos entrega una aproximación de la diversidad polínica, la que, sin embargo, puede estar sesgada y no representar fielmente la diversidad vegetal. Esto se debe a que, por lo general, el análisis morfológico del polen nos permite determinar tipos polínicos y no llegar al nivel taxonómico de especies. La identificación taxonómica de polen es el resultado de reconocer características morfológicas del grano, como, por ejemplo, tamaño, estructura general de la pared, tipo de apertura (poro, colpo o colporo) y ornamentaciones (e.g. tipo y número de espinas) (Heusser 1971, Punt et al. 2007). Salvo en el caso de las familias monotípicas (e.g. Aextoxicaceae), el análisis de microscopía óptica sólo permite identificar familias o tipos polínicos dentro de estas (Moore et al. 1991). Los resultados de nuestro estudio indican que los tipos polínicos más frecuentes son Asteraceae y Poaceae. Afortunadamente, es posible diferenciar dentro de las asteráceas taxa polínicos de diferente distribución altitudinal, tales como Asteraceae tipo Doniophyton, que caracteriza el piso andino. En contraste, la determinación de tipos polínicos dentro de la familia Poaceae resulta mucho más difícil, si no se cuenta con una colección de referencia completa de la zona en estudio (Weng et al. 2007).

Por otro lado, al existir una diferencia de producción de polen entre diferentes taxa (Hjelle 1997), el índice de ShannonWiener puede subestimar la diversidad polínica real (Matthias et al. 2015, Felde et al. 2016). Para determinar la diversidad vegetal mediante análisis de polen, se debe tomar en cuenta la equidad polínica (grado de igualdad de la distribución de la abundancia de los taxa polínicos) y su relación con la producción y dispersión (Tuomisto 2012, Matthias et al. 2015). En ambientes con una alta riqueza de familias, la identificación de estas utilizando el registro polínico podría ser adecuada para evaluar la diversidad vegetal (Jantz et al. 2014); sin embargo, en el caso de las familias Asteraceae, Poaceae, Fabaceae y Apiaceae, cuya riqueza específica es considerable, las estimaciones de diversidad vegetal exigen un reconocimiento a nivel específico (Cavieres et al. 2000). Estas diferencias de riqueza entre familias podrían redundar en el registro de patrones de diversidad polínica no siempre concordantes con los patrones altitudinales de diversidad vegetal.

Los resultados de concentración polínica no presentaron un patrón altitudinal muy definido; sin embargo, en las cuencas de El Yeso y El Volcán y para el Grupo I de Laguna del Viento, dicha variable alcanzó valores de los más altos a $2.100 \mathrm{~m} \mathrm{~s} . \mathrm{n}$. m. Esto podría deberse a la relativamente alta diversidad polínica registrada en dicho nivel y en los colindantes, además de una relativamente mayor estación de crecimiento en relación a los niveles altitudinales superiores. Adicionalmente, el microclima que puede generar el fondo de las quebradas, puede amortiguar las variaciones ambientales y genera un aumento de la riqueza y abundancia vegetal, situación que puede no concordar necesariamente con los patrones altitudinales esperados (Naiman et al. 1998). Finalmente, aunque la concentración y riqueza polínica actual se corresponda con la vegetación circundante, existen factores que pueden limitar esta relación, como, por ejemplo, la tasa de acumulación y tipos de sedimentos, la estabilidad de las laderas, la producción y dispersión de polen y las condiciones ambientales (e.g. los lugares secos pueden tener una menor concentración de polen que los húmedos) (Stuart et al. 2006, Zhao \& Sun 2010). 


\section{Polen SUPERficial Y SU SEÑal ANTRóPICA}

El polen superficial de una zona puede ser la representación de la estructura vegetal y del nivel de intervención del sitio (Colombaroli et al. 2013). En nuestros resultados, los grupos de menor altitud, caracterizados por la presencia de arbustos, presentaron en algunos casos más diversidad de taxa polínicos que otros, aunque es posible que dicha diversidad sea aún mayor y esté subestimada en el análisis. Lo anterior se debe a que, en algunos casos, en las muestras polínicas las herbáceas se ven mejor representadas que los arbustos, por su alta producción de polen y síndrome de polinización anemófila (Weng et al. 2007, Damialis et al. 2011). Cabe notar que, si bien la familia Poaceae es uno de los taxa polínicos más abundantes en todos los niveles, dada su alta producción de polen, también puede ser indicadora de sitios con influencias antropogénicas (Hicks 1993, Hjelle 1997). Esto se explica porque gran parte de las malezas pertenecen a esta familia (Matthei 1995). Los resultados de Laguna del Viento muestran que entre 2.300 y 2.500 m s. n. m. aumenta la presencia de Poaceae, lo que concuerda con su proximidad a centros mineros abandonados. Asimismo, en gran parte de la cordillera existen especies de la familia Poaceae introducidas, cuya mayor frecuencia se registra en rutas de arrieros y ganado que suben a la cordillera durante las veranadas. Otro ejemplo es la presencia de polen de Pinus en los transectos de El Volcán (2.232-2.436 m s. n. m.) y El Yeso (2.100-2.467 m s. n. m.). Al respecto, se sabe que Pinus radiata D. Don fue introducido en Chile en el año 1885 (Aztorquiza 1929, Estades \& Escobar 2005) y que a partir de 1974 se fomentó su cultivo, cuya distribución actual se extiende entre $33^{\circ}$ y $42^{\circ}$ S. Tanto las semillas como el polen de esta especie, registrada en más del $50 \%$ de las zonas habitadas, son dispersadas por el viento, por lo que, si bien no está en los puntos de muestreo, es posible que existan poblaciones de la especie cercanas a los transectos (Bustamante \& Simonetti 2005). La morfología polínica de Pinus, en especial las estructuras denominadas vesículas aéreas, hace que este tipo de polen pueda ser transportado a gran distancia por el viento, en comparación con tipos polínicos carentes de estas estructuras (Schwendemann et al. 2007).

\section{EL ROL DEL VIENTO}

Según datos de literatura, la distancia de dispersión del polen por medio del viento puede variar entre especies, según los factores ambientales y topográficos de la zona (Koenig \& Ashley 2003). Características climáticas como la velocidad y dirección de los vientos pueden influir en la tasa de sedimentación polínica en el suelo, ya sea disminuyendo el aporte de polen o contribuyendo a su transporte desde un piso de vegetación original a otros (Okubo \& Levin 1989). A pesar de que este estudio no contempla las características topoclimáticas (e.g. velocidad y dirección del viento) y geográficas de la zona, se identificó un patrón de distribución polínica dependiente del gradiente altitudinal, el cual puede presentar un desfase altitudinal de algunos metros respecto de los pisos de vegetación actual (e.g. Teillier et al. 2011) (Fig. 5). Esto se observó especialmente en el límite subandinoandino de Laguna del Viento, el que, según nuestros registros de polen, se ubicó $150 \mathrm{~m}$ por encima del límite descrito en la literatura. Los vientos en esta zona suelen tener un patrón de dirección desde el Norte y Noroeste (Rutllant \& Garreaud 2004), aunque depende de las características topográficas. Por otra parte, es conocido que en áreas cordilleranas los vientos de ladera son un factor importante y diario (Strahler \& Strahler 2013), consecutivo al calentamiento diferencial de las partes altas respecto de las bajas. Esto último ocurre sobre todo al amanecer, generando un flujo de viento ladera arriba. Generalmente, se observa un fenómeno opuesto durante la tarde, aunque en muchos casos tiene una menor intensidad. Factores como estos podrían explicar el desfase altitudinal entre la lluvia polínica y los pisos de vegetación, pues contribuyen a que la dispersión polínica sea más intensa hacia arriba.

\section{COMPARACIÓN CON OTROS ESTUDIOS}

Los datos de distribución polínica disponibles para el norte de Chile indican con claridad que la abundancia de los diferentes taxa tiende a variar gradualmente según el gradiente altitudinal (Maldonado et al. 2005, González et al. 2013, Collao-Alvarado et al. 2015, Zamora-Allendes 2015). Por ejemplo, la familia Amaranthaceae domina en pisos inferiores y va disminuyendo con el aumento de la altitud, mientras que Poaceae es dominante en pisos superiores y tiende a disminuir a menores altitudes en concordancia con la dominancia de estas familias en los distintos pisos de vegetación (Maldonado et al. 2005, González et al. 2013, Collao-Alvarado et al. 2015). Por otro lado, los datos de distribución polínica obtenidos en el sur del continente indican también una tendencia, pese a provenir principalmente de zonas boscosas (e.g. Heusser et al. 1995, Haberle \& Bennett 2001). En sus trabajos realizados en la Patagonia, Páez et al. (1994 y 1997) comparan de manera altitudinal los ensambles polínicos de dos transectos que incluyen ambas vertientes de los Andes, a los $38^{\circ}$ y $43^{\circ} \mathrm{S}$ (Argentina y Chile). Al oeste en altitudes bajas la presencia de tipos polínicos como Myrtaceae, Nothofagus y Eucryphia entre otros, indican la formación de Bosque Valdiviano (100300 m s.n.m.) y Nord Patagónico sin coníferas $(250-400$ m s.n.m.). A medida que incrementa en altitud la presencia de tipos polínicos como Myrtaceae, Loranthaceae, Nothofagus y Podocarpaceae, entre otras, describen las formaciones de 
Bosque Nord Patagónico con coníferas (400-700 m s.n.m.) y Bosque deciduo Subantártico. En cambio, al este de la cordillera, a medida que disminuye en altitud, desaparecen los tipos polínicos característicos de bosque y aparecen taxa polínicos característicos de El Monte (Chenopodiaceae, Larrea y Prosopis) y estepa patagónica (Poaceae y Cyperaceae), tendencia que se relaciona claramente con el clima de la zona. Esta tendencia en la distribución polínica altitudinal, tan clara a nivel familiar en los trabajos publicados para el norte y el sur, no fue registrada en esta contribución. Una posible explicación de ello es que en el centro de Chile las variaciones de composición florística están dadas por el reemplazo de especies dentro de un mismo género o familia (Cavieres et al. 2000, Teillier et al. 2011). Por ejemplo, en niveles altitudinales bajos de la zona central se pueden encontrar taxa polínicos que son compartidos con pisos altitudinales altos, como es el caso, en nuestros resultados, de Poaceae y Asteraceae. En el norte, en cambio, la familia Amaranthaceae no se repite en pisos de mayor altitud (González et al. 2013, Collao-Alvarado et al. 2015). Un patrón similar ocurre en el sur, donde taxa polínicos como Nothofagus, característico de pisos inferiores, no se encuentran en pisos de mayor altitud al hacer el análisis en un transecto a través de los Andes (Páez et al. 2001). Por otra parte, los trabajos citados incluyen transectos altitudinales o longitudinales de mucho mayor extensión, que abarcan todo o casi todo el espectro de vegetación presente. Por ejemplo, en los estudios efectuados en el norte de Chile los transectos parten en el límite inferior de la vegetación zonal y llegan casi hasta su límite superior, mientras que en los estudios del sur los transectos cruzan los Andes y atraviesan grandes formaciones vegetales muy contrastantes entre sí, tales como bosques siempreverdes, vegetación altoandina, estepa patagónica, etc.

\section{ConCLUSIÓN}

Este trabajo logra caracterizar la lluvia polínica de los Andes de Chile central en el gradiente altitudinal y determinar la diversidad polínica de esta zona. El análisis de polen del suelo a través de gradientes altitudinales, unido a las herramientas estadísticas de CONISS y psimpoll, permite una división de la señal polínica en pisos altitudinales. Esta señal es relativamente similar a la obtenida por fisonomía de la vegetación (pisos subandino, andino y altoandino); sin embargo, nuestro análisis falló en la subdivisión del piso andino en uno inferior y uno superior. Asimismo, se registraron algunas variaciones en la composición y límites altitudinales de los pisos entre cuencas, atribuibles a la dispersión polínica por el viento o a diferencias relacionadas con las características topográficas propias de cada cuenca (e.g. exposición, pendiente). En un caso, se observó una leve disminución de la riqueza y diversidad polínica según el gradiente altitudinal, cambio que puede ser menos perceptible que en la vegetación establecida, principalmente por la resolución taxonómica, producción de polen variable entre taxa y los factores de dispersión del polen.

Aun así, este trabajo es un ejemplo de que el uso del polen puede ser una herramienta útil para caracterizar las formaciones vegetales cuando no se dispone de datos de la flora establecida, ya sea presente o pasada. Finalmente, los datos presentados en este trabajo aportan a la caracterización de la vegetación de los Andes mediterráneos y su respuesta polínica, lo que constituye el único antecedente disponible para la zona andina en dicha latitud.

\section{AGRADECIMIENTOS}

Agradecemos a los proyectos Fondecyt 1140837, 1180413 y 1170408, a la Agrícola Santa Filomena, al Ing. Cristian Orrego y a los revisores que ayudaron a mejorar la calidad de este trabajo.

\section{REFERENCIAS}

Aceituno, P., Vidal, F. 1990. Variabilidad interanual en el caudal de ríos andinos de Chile Central en relación con la temperatura de la superficie del mar en el Pacífico Central. Revista Sociedad Ingeniería Hidráulica 5(1): 7-19.

Adam, D. P., Mehringer, P. J. 1975. Modern pollen surface samples-an analysis of subsamples. Journal of Research of the US Geological Survey 3(6): 733-736.

Arroyo, M.T.K., Armesto, J.J., Villagrán, C. 1981. Plant phenological patterns in the high Andean cordillera of central Chile. Journal of Ecology 69: 205-223.

Arroyo, M.T.K., Squeo, F., Armesto, J.J., Villagrán, C. 1988. Effects of aridity on plant diversity in the northern Chilean Andes: results of a natural experiment. Annals of the Missouri Botanical Garden 75: 55-78. DOI: 10.2307/2399466

Arroyo, M.T.K., Marquet, P.A., Marticorena, C., Cavieres, L.A., Squeo, F.A., Simonetti, J.A., Rozzi, R., Massardo, F. 2006. El hotspot chileno, prioridad mundial para la conservación. Diversidad de ecosistemas, ecosistemas terrestres. En: Comisión Nacional del Medio Ambiente (ed.), Diversidad de Chile: patrimonios y desafíos, pp. 94-97. Santiago, Chile.

Ashworth, A.C., Markgraf, V., Villagrán, C. 1991. Late Quaternary climatic history of the Chilean Channels based on fossil pollen and beetle analyses, with an analysis of the modern vegetation and pollen rain. Journal of Quaternary Science 6(4): 279-291. DOI: 10.1002/jqs.3390060403

Aztorquiza, O. 1929. Lota, antecedentes históricos con una monografía de la Compañía Minera e Industrial de Chile. 
Sociedad Imprenta y Litografía Concepción. 44 pp.

Bennett, K. 1996. Determination of the number of zones in a biostratigraphical sequence. New Phytologist 132: 155170.

Bustamante, R.O., Simonetti, J.A. 2005. Is Pinus radiata invading the native vegetation in central Chile? Demographic responses in a fragmented forest. Biological Invasions 7(2): 243-249. DOI: 10.1007/s10530-004-0740-5

Cañellas-Boltà, N., Rull, V., Vigo, J., Mercadé, A. 2009. Modern pollen-vegetation relationships along an altitudinal transect in the central Pyrenees (southwestern Europe). The Holocene 19(8): 1185-1200. DOI: 10.1177/0959683609345082

Cavieres, L.A., Arroyo, M.T.K. 1999. Tasa de enfriamiento adiabático del aire en el valle del Río Molina, Provincia de Santiago, Chile Central ( $\left.33^{\circ} \mathrm{S}\right)$. Revista Geográfica de Chile Terra Australis 44: 79-86.

Cavieres, L.A., Peñaloza, A., Kalin Arroyo, M. 2000. Altitudinal vegetation belts in the high-Andes of central Chile $\left(33^{\circ} \mathrm{S}\right)$. Revista Chilena de Historia Natural 73(2): 331-344.

Collao-Alvarado, K., Maldonado, A., González, L., Sandoval, A., De Porras, M.E., Zamora, A., Arancio, G. 2015. Estudio de la relación polen-vegetación actual en el Norte de Chile, en el transecto Pozo Almonte-Salar de Huasco (2015'S/6906'O). Gayana Botánica 72(1): 125-136. DOI: 10.4067/S0717-66432015000100015

Colombaroli, D., Beckmann, M., Knaap, W.O., Curdy, P., Tinner, W. 2013. Changes in biodiversity and vegetation composition in the central Swiss Alps during the transition from pristine forest to first farming. Diversity and Distributions 19(2): 157-170. DOI: 10.1111/j.1472-4642.2012.00930.x

Cruz, P., Schulze, C., Honeyman, P., Cabello, A. 2013. Quillaja saponaria Mol. En: Donoso, C. (ed.), Las especies arbóreas de los bosques templados de Chile y Argentina. Autoecología, Segunda edición, pp. 546-556. Marisa Cuneo Ediciones, Valdivia, Chile.

Cuevas, J. 1996. Hallazgo de Nassauvia lagascae (D. Don) Meigen var. lagascae (COMPOSITAE) a $4.300 \mathrm{~m}$ snm en los Andes de Santiago. Noticiario Mensual del Museo Nacional de Historia Natural 325: 38-39.

Cuevas, J.G., Le Quesne, C. 2006. Low vegetation recovery after short-term cattle exclusion on Robinson Crusoe Island. Plant Ecology 183(1): 105-124.

Damialis, A., Fotiou, C., Halley, J.M., Vokou, D. 2011. Effects of environmental factors on pollen production in anemophilous woody species. Trees 25(2): 253-264. DOI: 10.1007/s00468-010-0502-1

Di Castri, F., Hajek, E. 1976. Bioclimatología de Chile. Vicerrectoría Académica, Universidad Católica de Chile, Santiago. 163 pp.

Estades, C.F., Escobar, M.A.H. 2005. Los ecosistemas de las plantaciones de pino de la Cordillera de la Costa. En: Smith-Ramírez, C., Armesto, J., Valdovinos, C. (eds.), Historia, biodiversidad y ecología de los bosques costeros de Chile, pp. 600-616. Editorial Universitaria, Santiago, Chile.
Faegri, K., Iversen, J. 1989. Textbook of pollen analysis. Fourth edition. John Wiley \& Sons, New York. 328 pp.

Felde, V.A., Peglar, S.M., Bjune, A.E., Grytnes, J.A., Birks, H.J.B. 2016. Modern pollen-plant richness and diversity relationships exist along a vegetational gradient in southern Norway. The Holocene 26(2): 163-175. DOI: 10.1177/0959683615596843

Gómez-Casero, M.T., Hidalgo, P.J., García-Mozo, H., Domínguez, E., Galán, C. 2004. Pollen biology in four Mediterranean Quercus species. Grana 43(1): 22-30. DOI: 10.1080/00173130410018957

González-Silvestre, L., Maldonado, A., Núñez, L., Cartajena, I., Carrasco, C., De Souza, P. 2013. Condiciones paleovegetacionales y asentamientos humanos durante el Formativo temprano: análisis de polen del sitio Tulán-85 (1.530/1.260-460/420 años cal. A.C.), cuenca del Salar de Atacama. Chungará (Arica) 45(3): 387-410. DOI: 10.4067/S0717-73562013000300003

Gosling, W.D., Mayle, F.E., Tate, N.J., Killeen, T.J. 2009. Differentiation between Neotropical rainforest, dry forest, and savannah ecosystems by their modern pollen spectra and implications for the fossil pollen record. Review of Palaeobotany and Palynology 153(1): 70-85. DOI: $10.1016 /$ j.revpalbo.2008.06.007

Grimm, E. C. 1987. CONISS: a FORTRAN 77 program for stratigraphically constrained cluster analysis by the method of incremental sum of squares. Computers \& Geosciences 13(1): 13-35. DOI: 10.1016/00983004(87)90022-7

Haberle, S.G., Bennett, K.D. 2001. Modern pollen rain and lake mud-water interface geochemistry along environmental gradients in southern Chile. Review of Palaeobotany and Palynology 117(1-3): 93-107. DOI: 10.1016/S00346667(01)00079-3

Heusser, C. 1971. Pollen and spores of Chile: Modern types of Pteridophyta, Gymnospermae, and Angiospermae. Ediciones Universidad de Arizona, Tucson, Estados Unidos. $167 \mathrm{pp}$.

Heusser, C.J. 2003. Ice Age Southern Andes. A Chronicle of Paleoecological Events. Elsevier, Amsterdam. 240 pp.

Heusser, C.J., Denton, G.H., Hauser, A., Andersen, B.G., Lowell, T.V. 1995. Quaternary pollen records from the Archipiélago de Chiloé in the context of glaciation and climate. Andean Geology 22(1): 25-46.

Heusser, C.J., Heusser, L.E., Lowell, T.V. 1999. Paleoecology of the southern Chilean lake district-Isla Grande de Chiloé during middle-late Llanquihue glaciation and deglaciation. Geografiska Annaler: Series A, Physical Geography 81(2): 231-284. DOI: 10.1111/14680459.00058

Hicks, S. 1993. Pollen evidence of localized impact on the vegetation of northernmost Finland by hunter-gatherers. Vegetation History and Archaeobotany 2(3): 137-144.

Hidalgo, P.J., Galán, C., Domínguez, E. 2003. Male phenology of three species of Cupressus: correlation with airborne 
pollen. Trees 17(4): 336-344. DOI: 10.1007/s00468002-0243-x

Hjelle, K.L. 1997. Relationships between pollen and plants in human-influenced vegetation types using presenceabsence data in western Norway. Review of Palaeobotany and Palynology 99(1): 1-16. DOI: 10.1016/S00346667(97)00041-9

Jackson, S.T., Williams, J.W. 2004. Modern analogs in Quaternary paleoecology: here today, gone yesterday, gone tomorrow?. Annual Review of Earth and Planetary Sciences 32: 495-537. DOI: 10.1146/annurev. earth.32.101802.120435

Jantz, N., Homeier, J., Behling, H. 2014. Representativeness of tree diversity in the modern pollen rain of Andean montane forests. Journal of Vegetation Science 25(2): 481-490. DOI:10.1111/jvs.12105

Koenig, W.D., Ashley, M.V. 2003. Is pollen limited? The answer is blowin' in the wind. Trends in Ecology \& Evolution 18(4): 157-159. DOI: 10.1016/S0169-5347(03)00034-X

Kuentz, A., Galán de Mera, A., Ledru, M.P., Thouret, J.P. 2007. Phytogeographical data and modern pollen rain of the puna belt in southern Peru (Nevado Coropuna, Western Cordillera). Journal of Biogeography 34(10): 1762-1776. DOI: 10.1111/j.1365-2699.2007.01728.x

Kuoppamaa, M., Goslar, T., Hicks, S. 2009. Pollen accumulation rates as a tool for detecting land-use changes in a sparsely settled boreal forest. Vegetation History and Archaeobotany 18(3): 205-217. DOI: 10.1007/s00334008-0205-3.

Lagos, D.G. 2017. Estudio de calibración polen-vegetación del Parque Nacional Laguna del Laja (Chile central). Tesis para optar al Título de Profesor de Ciencias Naturales y Biología. Escuela de Educación, Universidad de Concepción. Los Ángeles. $58 \mathrm{pp}$.

Luebert, F., Pliscoff, P. 2006. Sinopsis bioclimática y vegetacional de Chile. Editorial Universitaria, Santiago. 316 pp.

Lupo, L. 1998. Estudio sobre la lluvia polínica actual y la evolución del paisaje a través de la vegetación durante el Holoceno en la cuenca del río Yavi. Borde oriental de la puna, noroeste argentino. Tesis Doctoral (Inédito). Fakultat fur Geschichts-und Geowissenschaften, Universitat Bamberg. Alemania. $87 \mathrm{pp}$.

Luzio, W., Seguel, O., Casanova, M. 2010. Suelos de la Zona Mediterránea Árida ( $32^{\circ} 00^{\prime}$ LS hasta $37^{\circ} 45^{\prime}$ LS). En: Luzio, W. (ed.), Suelos de Chile, pp. 125-194. Ediciones Universidad de Chile, Santiago, Chile.

Magurran, A.E. 1988. Ecological diversity and its measurement. Editorial Croom Helm, London. 179 pp.

Maldonado, A., Betancourt, J.L., Latorre, C., Villagran, C. 2005. Pollen analyses from a $50000-y r$ rodent midden series in the southern Atacama Desert ( $25^{\circ} 30^{\prime} \mathrm{S}$ ). Journal of Quaternary Science 20(5): 493-507. DOI: 10.1002/ jqs.936

Mancini, M.V. 1998. Vegetational changes during Holocene in the Extra-Andean Patagonia, Santa Cruz Province, Argentina.
Palaeogeography, Palaeoclimatology, Palaeoecology 138(1-4): 207-219.

Markgraf, V., D'Antoni, H.L. 1978. Pollen flora of Argentina. University of Arizona Press, Arizona. 208 pp.

Markgraf, V., Webb, R.S., Anderson, K.H., Anderson, L. 2002. Modern pollen/climate calibration for southern South America. Palaeogeography, Palaeoclimatology, Palaeoecology 181(4): 375-397. DOI; 10.1016/S00310182(01)00414-X

Matthei, Ó. 1995. Manual de las malezas que crecen en Chile. Alfabeta Impresores, Santiago. 545 pp.

Matthias, I., Semmler, M.S.S., Giesecke, T. 2015. Pollen diversity captures landscape structure and diversity. Journal of Ecology 103(4): 880-890. DOI: 10.1111/13652745.12404

Meltsov, V., Poska, A., Odgaard, B.V., Sammul, M., Kull, T. 2011. Palynological richness and pollen sample evenness in relation to local floristic diversity in southern Estonia. Review of Palaeobotany and Palynology 166(3): 344-351. DOI: 10.1016/j.revpalbo.2011.06.008

Montade, V., Peyron, O., Favier, C., Francois, J.P., Haberle, S.G. 2019. A pollen-climate calibration from western Patagonia for palaeoclimatic reconstructions. Journal of Quaternary Science 34(1): 76-86. DOI: 10.1002/jqs.3082

Moore, P.D., Webb, J.A., Collinson, M.E. 1991. Pollen analysis. Blackwell Scientific, Oxford. 216 pp.

Muñoz, A.A., Arroyo, M.T. 2006. Pollen limitation and spatial variation of reproductive success in the insect-pollinated shrub Chuquiraga oppositifolia (Asteraceae) in the Chilean Andes. Arctic, Antarctic, and Alpine Research 38(4): 608-613. DOI: 10.1657/1523-0430(2006)38[608:PLASVO]2.0.CO;2

Muñoz-Schick, M., Moreira-Muñoz, A., Villagrán, C., Luebert, F. 2000. Caracterización florística y pisos de vegetación en los Andes de Santiago, Chile Central. Boletín Museo Nacional de Historia Natural 49: 9-50.

Naiman, R.J., Fetherston, K.L., McKay, S.J., Chen, J. 1998. Riparian Forests. In: Naiman, R.J., Bilby, R.E. (eds.), River Ecology and Management, pp. 289-323. Springer, New York.

Okubo, A., Levin, S.A. 1989. A theoretical framework for data analysis of wind dispersal of seeds and pollen. Ecology 70(2): 329-338. DOI: 10.2307/1937537

Oswald, W.W., Brubaker, L.B., Hu, F.S., Gavin, D.G. 2003. Pollen vegetation calibration for tundra communities in the Arctic Foothills, northern Alaska. Journal of Ecology 91(6): 1022-1033. DOI: 10.1046/j.1365-2745.2003.00823.x

Páez, M.M., Villagrán, C., Carrillo, R. 1994. Modelo de la dispersión polínica actual en la región templada chileno-argentina de Sudamérica y su relación con el clima y la vegetación. Revista Chilena de Historia Natural 67(4): 417-434.

Páez, M.M., Villagrán, C., Stutz, S., Hinojosa, F., Villa, R. 1997. Vegetation and pollen dispersal in the subtropicaltemperate climatic transition of Chile and Argentina. Review of Palaeobotany and Palynology 96(1-2): 169181. DOI: 10.1016/S0034-6667(96)00039-5 
Páez, M.M., Schäbitz, F., Stutz, S. 2001. Modern pollen-vegetation and isopoll maps in southern Argentina. Journal of Biogeography 28(8): 997-1021. DOI: 10.1046/j.13652699.2001.00616.x

Punt, W., Hoen, P.P., Blackmore, S., Nilsson, S., Le Thomas, A. 2007. Glossary of pollen and spore terminology. Review of Palaeobotany and Palynology 143(1-2): 1-81.

Quintanilla, V. 1980. Observaciones fitogeográficas en la alta cordillera de Santiago. Revista Geográfica Terra Australis 24: 3-14.

Reese, C.A., Liu, K.B. 2005. Interannual variability in pollen dispersal and deposition on the tropical Quelccaya Ice Cap. The Professional Geographer 57(2): 185-197.DOI: 10.1111/j.0033-0124.2005.00471.x

Rodríguez, R., Marticorena, C., Alarcón, D., Baeza, C., Cavieres, L., Finot, V.L., Fuentes, N., Kiessling, A., Mihoc, M., Pauchard, A., Ruiz, E., Sánchez, P., Marticorena, A. 2018. Catálogo de las plantas vasculares de Chile. Gayana Botánica 75(1): 1-430. DOI: 10.4067/S0717-66432018000100001

Rozzi, R., Molina, J., Miranda, P. 1989. Microclima y periodos de floración en laderas de exposición ecuatorial y polar en los Andes de Chile central. Revista Chilena de Historia Natural 62(1): 75-84.

Rutllant, J., Garreaud, R. 2004. Episodes of Strong Flow down the Western Slope of the Subtropical Andes. Monthly Weather Review 132: 611-622. DOI: 10.1175/1520-0493(2004)132<0611:EOSFDT>2.0.CO;2

Sarricolea, P., Herrera-Ossandon, M., Meseguer-Ruiz, Ó. 2017. Climatic regionalisation of continental Chile. Journal of Maps 13 (2): 66-73. DOI: 10.1080/17445647.2016.1259592.

Schwendemann, A.B., Wang, G., Mertz, M.L., Mcwilliams, R.T., Thatcher, S.L., Osborn, J. M. 2007. Aerodynamics of saccate pollen and its implications for wind pollination. American Journal of Botany 94(8): 1371-1381. DOI 10.3732/ajb.94.8.1371

Smith-Ramírez, C., Armesto, J.J., Valdovinos, C. (eds.). 2005. Historia, biodiversidad y ecología de los bosques costeros de Chile. Editorial Universitaria, Santiago. 708 pp.

Solomon, E., Berg, L., Martin, D. 2008. Biología (Octava edición). Mc Graw Hill, México. 1400 pp.

Squeo, F.A., Veit, H., Arancio, G., Gutiérrez, J.R., Arroyo, M.T.K., Olivares, N. 1993. Spatial heterogeneity of high mountain vegetation in the Andean desert zone of Chile. Mountain Research and Development 13(2): 203-209.

Stockmarr, J.A. 1971. Tablets with spores used in absolute pollen analysis. Pollen spores 13: 615-621.

Strahler, A.H., Strahler, A.N. 2013. Introducing physical geography. Wiley, New York. 672 pp.
Stuart, G., Gries, C., Hope, D. 2006. The relationship between pollen and extant vegetation across an arid urban ecosystem and surrounding desert in Southwest USA. Journal of Biogeography 33(4): 573-591. DOI:10.1111/ j.1365-2699.2005.01334.x

Sugita, S., Hicks, S., Sormunen, H. 2010. Absolute pollen productivity and pollen-vegetation relationships in northern Finland. Journal of Quaternary 25(5): 724-736. DOI: $10.1002 /$ jqs.1349

Teillier, S., Hoffmann, A.J., Saavedra, F., Pauchard, L. 1994. Flora del Parque Nacional El Morado (Región Metropolitana, Chile). Gayana Botánica 51: 13-47.

Teillier, S., Marticorena, A., Niemeyer, H. M. 2011. Flora andina de Santiago: guía para la identificación de las especies de las cuencas del Maipo y del Mapocho. Imprenta Puntografix, Santiago. 480 pp.

Trivi de Mandri, M.E., Burry, L.S., D’Antoni, H.L. 2006. Dispersióndepositación del polen actual en Tierra del Fuego, Argentina. Revista Mexicana de Biodiversidad 77(1): 8995. DOI: 10.22201/ib.20078706e.2006.001.322

Tuomisto, H. 2012. An updated consumer's guide to evenness and related indices. Oikos 121(8): 1203-1218. DOI: 10.1111/j.1600-0706.2011.19897.x

Villagrán, C. 1980. Vegetationsgeschichtliche und pflanzensoiziologische Untersuchengen im Vicente Pérez Rosales Nationalpark, Chile. Dissertationes Botanicae 54: 1-165.

Villagrán, C., Kalin Arroyo, M.T., Marticorena, C. 1983. Efectos de la desertización en la distribución de la flora andina de Chile. Revista Chilena de Historia Natural 56(2): 137-157.

Weng, C., Bush, M.B., Silman, M.R. 2004. An analysis of modern pollen rain on an elevational gradient in southern Peru. Journal of Tropical Ecology 20(1): 113-124. DOI: 10.1017/ S0266467403001068

Weng, C., Hooghiemstra, H., Duivenvoorden, J.F. 2007. Response of pollen diversity to the climate-driven altitudinal shift of vegetation in the Colombian Andes. Philosophical Transactions of the Royal Society B Biological Sciences 362(1478): 253-262. DOI: 10.1098/rstb.2006.1985

Zamora-Allendes, A.F. 2015. Cuantificación del gradiente climático actual en la vertiente occidental de los Andes (entre 18-25 S) a partir de madrigueras de roedores. Tesis de Maestría. Facultad de Biología, Universidad de La Serena. La Serena, Chile. 176 pp.

Zhao, Y., Sun, Q.F. 2010. Reliability of pollen concentration as the indicator of effective moisture in arid and semi-arid regions of China. Journal of Arid Environments 74(3): 423-427. DOI: 10.1016/j.jaridenv.2009.09.012

Received: 27.08.2018

Accepted: 02.10.2019 